\title{
Functional Magnetic Resonance Imaging of Delay and Trace Eyeblink Conditioning in the Primary Visual Cortex of the Rabbit
}

\author{
Michael J. Miller, ${ }^{1,2}$ Craig Weiss, ${ }^{4}$ Xiaomu Song, ${ }^{2,3}$ Gheorghe Iordanescu, ${ }^{2,3}$ John F. Disterhoft, ${ }^{4}$ and Alice M. Wyrwicz ${ }^{2,3}$ \\ ${ }^{1}$ Biomedical Engineering Department, Northwestern University, Evanston, Illinois 60201, ${ }^{2}$ Center for Basic Magnetic Resonance Research, Evanston \\ Northwest Healthcare Research Institute, Evanston, Illinois 60201, and Departments of ${ }^{3}$ Radiology and ${ }^{4}$ Physiology, Feinberg Medical School, \\ Northwestern University, Chicago, Illinois 60611
}

\begin{abstract}
The primary sensory cortices have been shown in recent years to undergo experience- and learning-related plasticity under a variety of experimental circumstances. In this study, we used functional magnetic resonance imaging (fMRI) in parallel with both delay and trace eyeblink conditioning to image the learning-related functional activation within the primary visual cortex (V1) of awake, behaving rabbits. We expected that the differing level of forebrain dependence between these two conditioning paradigms should produce a differential blood oxygenation level-dependent (BOLD) functional response in V1. Our results showed a significant expansion of activated volume within $\mathrm{V} 1$, particularly early in learning, after training with the more cognitively demanding trace paradigm. In contrast, the simpler delay paradigm produced an increase in the magnitude of the BOLD response in activated voxels, but no significant change in activated volume. No accompanying learning-related changes were observed in the primary somatosensory cortex, which mediates the unconditioned stimulus. These results suggest that the recruitment of additional neurons within V1 is necessary to support the more demanding memory imposed by the trace interval. To our knowledge, this work is the first functional imaging study to compare directly trace and delay eyeblink conditioning in an animal model.
\end{abstract}

Key words: fMRI; eyeblink conditioning; visual cortex; rabbit; learning; memory

\section{Introduction}

The contribution of the sensory cortices to learning and memory in classical conditioning has received increased attention recently, although much previous work has focused on the cerebellum and hippocampus. Classical conditioning of the rabbit eyeblink response has long been used to investigate learning and memory (Gormezano et al., 1983). This associative task pairs a neutral conditioning stimulus (CS) with a salient unconditioned stimulus (US) that evokes a behavioral response. After repeated paired presentations, the subject learns to associate the stimuli and exhibits a conditioned response (CR) before the onset of the US. Combined with lesions or electrophysiological recordings, eyeblink conditioning (EBC) provides a well controlled paradigm for investigating the neurobiological mechanisms of learning and memory (Lavond et al., 1993; Thompson and Kim, 1996; Weiss et al., 2006). More recently, functional imaging techniques have allowed the simultaneous noninvasive examination of large vol-

Received Dec. 19, 2007; revised March 4, 2008; accepted March 28, 2008.

This work was supported by National Institutes of Health Grants R01 MH58912 and S10 RR15685 (A.M.W.) and National Research Service Award Predoctoral Fellowship F31 MH65085 (M.J.M.). We thank Daniil Aksenov for his input and comments.

Correspondence should be addressed to Alice M. Wyrwicz, Center for Basic Magnetic Resonance Research, Evanston Northwest Healthcare Research Institute, 1033 University Place, Suite 100, Evanston, IL 60201. E-mail: a-wyrwicz@northwestern.edu.

DOI:10.1523/JNEUROSCI.5622-07.2008

Copyright $\odot 2008$ Society for Neuroscience $\quad 0270-6474 / 08 / 284974-08 \$ 15.00 / 0$ umes of the brain, and both positron emission tomography (PET) and functional magnetic resonance imaging (fMRI) studies have investigated EBC (Schreurs and Alkon, 2001).

An advantage of EBC is that easily controlled manipulations of the stimulus paradigm can engage different neuronal circuitry. For example, delay conditioning, in which the CS and US overlap and coterminate, requires only the brainstem and cerebellum to learn and retain the association (Norman et al., 1977; Mauk and Thompson, 1987). More cognitively complex trace conditioning, in which a stimulus-free interval separates the CS and US, requires the hippocampus and other forebrain structures (Solomon et al., 1986; Moyer et al., 1990; Weible et al., 2000).

Once thought to be passive receivers of sensory information, the primary sensory cortices have exhibited plasticity within a variety of experimental contexts. Classical conditioning (Bakin and Weinberger, 1990) and frequency discrimination training (Recanzone et al., 1993) have produced receptive field plasticity in the auditory cortex. Amputation (Merzenich et al., 1984), retinal lesions (Kaas et al., 1990), and sensory discrimination training (Recanzone et al., 1992) have produced cortical reorganization. These studies and others have demonstrated that learning and experience can modulate the sensory cortices, and suggest a potential role for these regions in memory formation and storage. However, no imaging study has explicitly compared the functional activation during trace and delay EBC in a conscious animal model. 
Previously, we demonstrated reliable detection of functional activation in the visual system of the awake, behaving rabbit using fMRI (Wyrwicz et al., 2000) and imaged learning-related changes in the rabbit cerebellum during delay EBC (Miller et al., 2003). This study uses fMRI in parallel with delay and trace EBC to investigate learning-related changes in the rabbit primary visual cortex (V1). As this region mediates perception of the visual CS, we expected it to exhibit learning-related plasticity. Moreover, the differential dependence of trace and delay conditioning on forebrain structures could be expected to produce distinct patterns of functional activation in V1 with learning. We analyze the changes in both activated volume and blood oxygenation leveldependent (BOLD) response within V1 before, during, and after learning. For comparison, we also examine the primary somatosensory cortex (S1), which mediates the US. Our results indicate that both paradigms produce distinct learning-related changes in V1 without accompanying changes in S1.

\section{Materials and Methods}

Animal preparation. The rabbits were surgically prepared for the experiments as described previously (Wyrwicz et al., 2000), which included securing four nylon restraining bolts to the skull with nylon skull screws and dental cement. The headbolt was stereotaxically implanted in the horizontal plane to position the skull with lambda $1.5 \mathrm{~mm}$ below bregma, and was used to secure the radiofrequency (RF) coil and the rabbit's head in the same position to obtain a constant imaging angle and slice positioning among subjects. Each subject was habituated to the imaging environment before the experiments. All procedures were performed under National Institutes of Health guidelines, and Evanston Northwestern Healthcare Research Institute and Northwestern University Institutional Animal Care and Use Committee approved protocols.

Ten Dutch belted female rabbits were used in this study $(2-3 \mathrm{~kg})$. Five animals were trained with the delay paradigm, and five with the trace paradigm. An additional eight rabbits were prepared for MR imaging, but were unable to habituate sufficiently to the MRI environment, as determined by their movement during initial images acquired before the conditioning experiments.

fMRI data collection. Imaging was performed using a Bruker (Billerica, MA) Avance 4.7 T imaging spectrometer operating at a proton frequency of $200 \mathrm{MHz}$. This system was equipped with an Oxford horizontal magnet and an Acustar actively shielded gradient coil assembly with a clear bore of $15 \mathrm{~cm}$. A flat, circular surface coil ( $60 \mathrm{~mm}$ diameter) was used for $\mathrm{RF}$ transmission and reception. A multislice, single-shot gradient echoplanar imaging pulse sequence, with a repetition time (TR) of $2 \mathrm{~s}$ and an echo time (TE) of $21 \mathrm{~ms}$, was used to map brain activation. Coronal images in a plane perpendicular to the surface coil were collected from eight slices ( $1.0 \mathrm{~mm}$ thickness) using a $64 \times 80$ matrix size reconstructed to $64 \times 128$, and a $48 \times 48 \mathrm{~mm}$ field of view (FOV), corresponding to an in-plane resolution of $750 \times 375 \mu \mathrm{m}$. The slice positioning was aligned with respect to a clear anatomical landmark (the caudal edge of the cerebellar vermis) as viewed in a midsagittal image of each subject on each day to assure reproducibility between experiments. Thirty-five images were collected per trial. High-resolution images for anatomical identification $(256 \times 256$ matrix, $48 \times 48 \mathrm{~mm}$ FOV, $188 \times 188 \mu \mathrm{m}$ in-plane resolution) were also obtained using a multislice gradient echo sequence ( $1.0 \mathrm{~mm}$ slice thickness; TR, $1 \mathrm{~s}$; TE, $10 \mathrm{~ms}$ ).

Stimulus presentation and eyeblink measurement. The eyelid of the right eye was held open to measure movement of the nictitating membrane (NM) and to ensure activation by the visual CS. The eyelid of the left eye was covered during experiments. Rabbits were provided with earplugs during the experiments to reduce noise from the gradients. The visual CS was delivered by a $2 \times 2$ array of green light-emitting diodes $(2 \times 2 \mathrm{~mm}$, separated by $8 \mathrm{~mm}$ along each axis). The US consisted of a 3 psi airpuff supplied by compressed air and controlled by a regulator and solenoid valve, using a system described previously (Li et al., 2003). Both stimuli were delivered to the right eye. NM movement was measured with a fiber-optic-based infrared reflectance sensor (Miller et al., 2005). The fiber-optic probe was positioned $\sim 1 \mathrm{~cm}$ from the cornea. The durations of the CS and US were 850 and $100 \mathrm{~ms}$, respectively, for the delay paradigm. For the trace paradigm, the durations of the CS and US were 250 and $100 \mathrm{~ms}$, respectively, with a $500 \mathrm{~ms}$ stimulus-free trace interval. Trials were given every $70 \mathrm{~s}$, with a single period of stimulation in each trial. To prevent the animals from potentially using the gradient pulses as a timing cue, the timing of the stimulus presentation during each trial was randomized within an interval of up to three $2 \mathrm{~s}$ TR periods after the acquisition of 13 baseline images. fMRI data were realigned with respect to the stimulus presentation before averaging.

A CR was defined as a change in the voltage from the detector that was $4 \mathrm{SD}$ greater than the mean baseline amplitude, and occurred at least 35 $\mathrm{ms}$ after onset of the CS, but before the US. Eyeblink data were sampled at $300 \mathrm{~Hz}$. Each subject received two sessions (40 trials per session) of "pseudoconditioning" trials, consisting of random, unpaired CS and US presentations to measure the BOLD response to each individual stimulus before learning. Subjects then received training sessions (40 trials/session) consisting of paired-stimulus trials until they reached a learning level of $80 \%$ CRs. After reaching $80 \%$ CRs, the animals received one session of CS alone ("memory") trials to more directly compare the response to the CS after training to that observed in CS alone trials during pseudoconditioning. The animals were initially trained in the magnet but without the gradient noise present until they began to exhibit CRs to facilitate learning, and were then imaged for all subsequent sessions.

fMRI data analysis. The fMRI data were registered using a twodimensional affine method implemented in the National Laboratory of Medicine Insight Toolkit (Ibanez and Schroeder, 2005), and then detrended using a multitaper method-based algorithm (Mitra and Pesaran, 1999) to remove baseline drift. The detrending and subsequent processing were implemented using Matlab (The MathWorks, Natick, MA). After detrending, all trials were inspected for any remaining head movement, and trials showing movement were excluded from the analysis; the remaining trials were then averaged. The averaged fMRI data were denoised using a spatiotemporal Bayesian wavelet shrinkage method with a Haar wavelet (Song et al., 2006). Activated voxels were then detected in the denoised data using an unsupervised one-class support vector machine-based algorithm as described previously (Song and Wyrwicz, 2007). After processing, the data were analyzed to measure mean activated volume within $\mathrm{V} 1$ and the mean BOLD temporal response of activated voxels at learning levels of 40 and $80 \%$ CRs, as well as for pseudoconditioning CS and US trials and CS-alone memory trials after training. The volume of activation in each case was calculated by measuring the number of active voxels within the volume of V1 examined in this experiment, on the contralateral side of each subject lateral to the splenial sulcus and extending from $\sim 6-10 \mathrm{~mm}$ posterior to bregma (Hughes and Vaney, 1982; Choudhury, 1987), which represents approximately the anterior $4 \mathrm{~mm}$ of V1. The mean BOLD temporal responses of the active voxels in this region were subsequently averaged across subjects at each stage to generate a mean response for each paradigm at 40 and $80 \%$ CRs, as well as for pseudoconditioning CS and US and memory trials. For comparison, slices containing the eye region of the somatosensory cortex, which mediates reception of the US, were also identified $\sim 2-4 \mathrm{~mm}$ posterior to bregma, and an identical analysis was performed on this region.

\section{Results}

The rabbits used in this study adapted well to the restraint and imaging environment and showed no obvious sign of movement or struggling during the experiments. The delay-conditioned rabbits attained a level of $40 \%$ CRs in $4.2 \pm 0.86$ sessions and a level of $80 \%$ CRs in $5.8 \pm 0.66$ sessions. The trace-conditioned rabbits attained a level of $40 \%$ CRs in $5.6 \pm 1.3$ sessions, and a level of $80 \%$ CRs in $8.0 \pm 1.5$ sessions. An ANOVA of paradigm by criterion level indicated a significant main effect of the criterion level, i.e., significantly more sessions were required to reach the $80 \%$ criterion than the $40 \%$ criterion $\left(F_{(1,8)}=72.7 ; p<\right.$ 0.0001 ) when combining both the delay and trace-conditioned rabbits. The main effect of trace versus delay was not significantly 


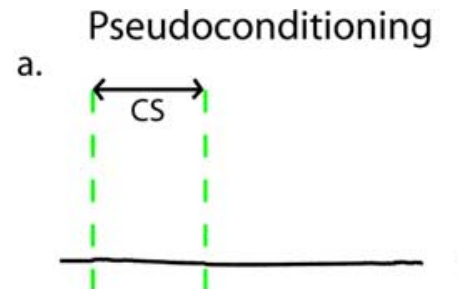

b.

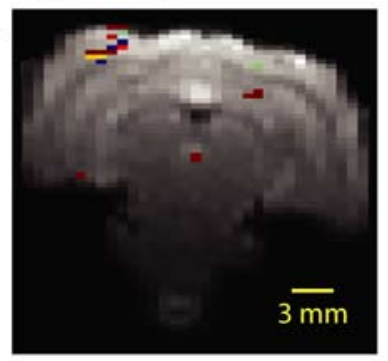

C.

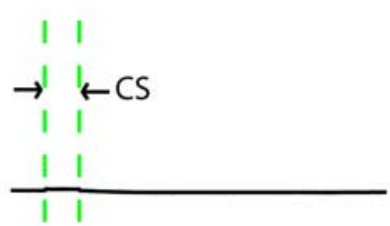

d.

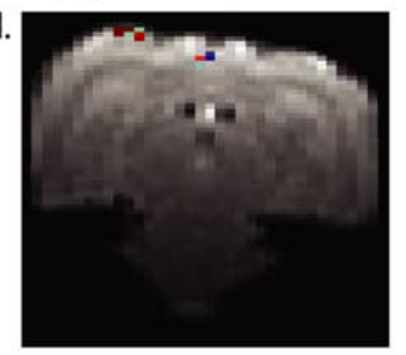

$40 \%$ CRs
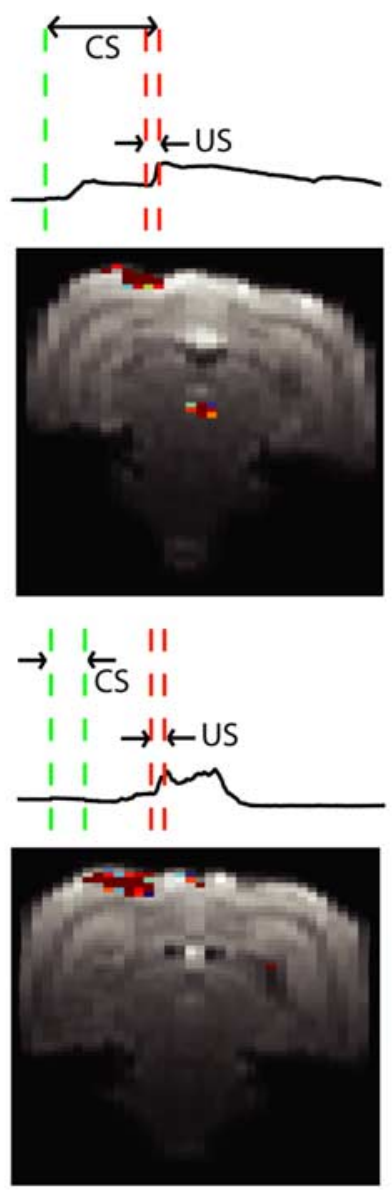

$80 \%$ CRs
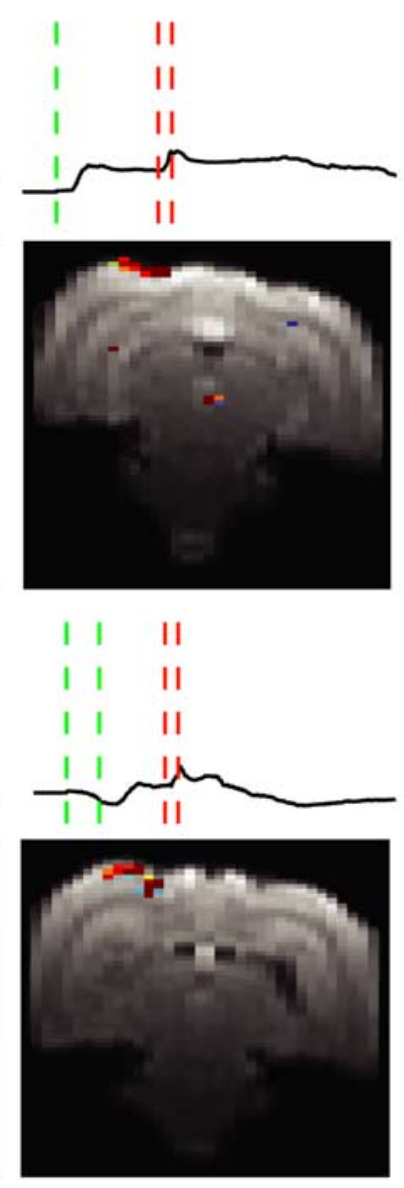

Memory
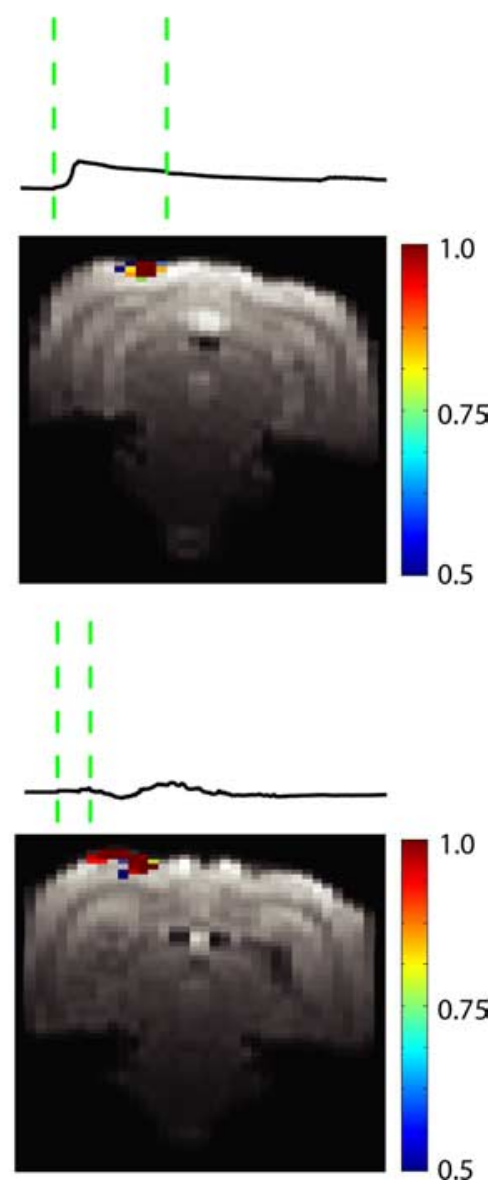

Figure 1. Behavioral responses and functional images of V1. $\boldsymbol{a}-\boldsymbol{d}$, Behavioral eyeblink responses for delay $(\boldsymbol{a})$ and trace $(\boldsymbol{c})$ conditioning and corresponding functional images obtained during delay $(\boldsymbol{b})$ and trace $(\boldsymbol{d})$ conditioning. The behavioral responses represent the average of trials within a single session. Note the development of CRs with training, indicating successful acquisition of the paradigms. The functional images show a slice from a representative subject containing the primary visual cortex, which at this level extends laterally for $\sim 3-5 \mathrm{~mm}$ from the splenial sulcus. Note the significant increase in activated area in V1 during trace conditioning, in contrast to delay conditioning. The color map in this case reflects the probability of activation, and is not related to the magnitude of the BOLD response.

different, although the trend was in the expected direction. This result likely reflects the initial hurdle of overcoming the distraction associated with the gradient noise in the MRI environment.

Figure 1 compares the behavioral eyeblink responses and functional images acquired during delay and trace conditioning from a representative single subject for each paradigm during pseudoconditioning CS trials, training (40 and $80 \%$ CRs), and CS-alone memory trials after training. The behavioral responses (Fig. 1a,c) were averaged across each session. The images (Figs. $1 b, d)$ show the corresponding functional activation observed in a representative slice containing the visual cortex $(\sim 10 \mathrm{~mm}$ posterior to bregma) for both the delay and trace subject.

As expected, the rabbits showed no behavioral response to the CS during pseudoconditioning sessions in either paradigm. During training the rabbits began to show CRs, providing clear evidence of a learned behavioral response for both paradigms. The responses early in learning in the delay paradigm (Fig. 1a) were visibly more robust than those from the more difficult trace paradigm (Fig. 1c), but both paradigms produced robust responses by the late stages of training. Furthermore, the rabbits in both paradigms exhibited CRs in response to the CS even in the absence of the US after training, whereas there was no response to CS trials during pseudoconditioning. The mean $( \pm$ SE) percent- age of trials showing CRs during the CS alone memory session was $94 \pm 2.3 \%$ for the delay group and $87 \pm 5.0 \%$ for the trace group, indicating that little extinction occurred during these sessions. A $t$ test indicated no significant difference in performance between the groups.

The functional images (Figs. 1b,d) reveal distinct differences between trace and delay conditioning in terms of the activated area within contralateral V1 corresponding to these behavioral changes. Contralateral activation is expected in response to a unilateral visual stimulus because the retinal projections in the rabbit are $\sim 90-95 \%$ crossed, as determined by anatomical (Giolli and Guthrie, 1969; Chihara, 1982; Steele et al., 1987) and physiological (Thompson et al., 1950; Murphy and Berman, 1979; Hughes and Vaney, 1982; Choudhury, 1987) methods. For both paradigms, a single cluster of activated voxels can be seen, located well within the boundaries of the primary visual cortex, which at this level extends laterally from the splenial sulcus near the midline for $\sim 3-5 \mathrm{~mm}$ (Thompson et al., 1950; Hughes and Vaney, 1982; Choudhury, 1987). In the case of delay conditioning, little change in the activated area can be observed either during training or in memory trials. In contrast, the activated area expands visibly during training in the case of trace condi- 


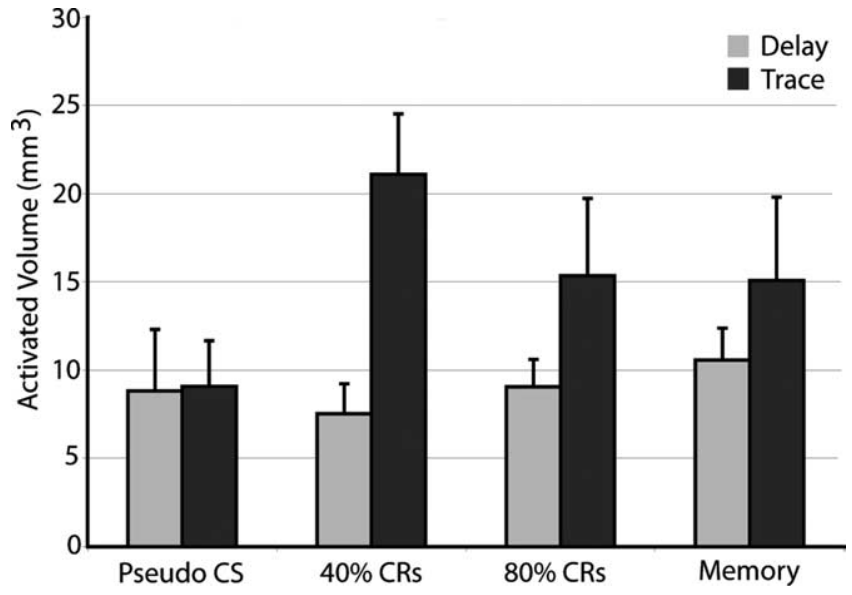

Figure 2. Activated volume in V1. The mean ( $n=5$ for each paradigm) activated volume in V1 for both trace and delay conditioning is shown during pseudoconditioning CS trials, at 40 and $80 \%$ CRs, and during CS alone memory trials after training. Note that delay conditioning produces no significant change in volume with learning, whereas trace conditioning produces a significant $(p<0.05)$ expansion of activated volume at $40 \%$ CRs, with respect to both pseudoconditioning CS trials and delay conditioning at 40\% CRs. Error bars represent SEM.

tioning, and then decreases during memory trials. No activation was observed in V1 during pseudoconditioning US trials.

Figure 2 shows the averaged results for the activated volume within the region of the visual cortex imaged in this study. Delay subjects exhibited an activated volume of $8.8 \pm 3.5 \mathrm{~mm}^{3}$ (mean $\pm \mathrm{SE}$ ) during pseudoconditioning CS trials, $7.5 \pm 1.7$ $\mathrm{mm}^{3}$ at $40 \%$ CRs, $9.1 \pm 1.6 \mathrm{~m}^{3}$ at $80 \% \mathrm{CRs}$, and $11 \pm 1.8 \mathrm{~mm}^{3}$ during memory trials. In contrast, trace subjects exhibited activated volumes of $9.1 \pm 2.6 \mathrm{~mm}^{3}$ during pseudoconditioning CS trials, $21.1 \pm 3.4 \mathrm{~mm}^{3}$ at $40 \% \mathrm{CRs}, 15.3 \pm 4.4 \mathrm{~mm}^{3}$ at $80 \% \mathrm{CRs}$, and $15.1 \pm 4.7 \mathrm{~mm}^{3}$ during memory trials. A repeated-measures ANOVA with paradigm as a factor revealed a significant interaction $\left(F_{(2,16)}=3.97 ; p=0.040\right)$ between the training paradigm (trace/delay) and the three stages of training (pseudoconditioning, 40 and 80\%). A post hoc ANOVA for each group separately indicated that the trace-conditioned group exhibited a significant difference $\left(F_{(2,8)}=5.2 ; p=0.035\right)$ among the three stages, but the delay-conditioned group did not. Post hoc Fisher's least significant difference (LSD) tests indicated that the difference occurred between the baseline pseudoconditioning session and the session at $40 \%$ CRs $(p=0.010)$. Post hoc $t$ tests were used to compare the activated volumes for delay and trace conditioning at each stage of the experiment. These comparisons revealed a significant increase in activated volume for trace subjects versus delay subjects at $40 \% \operatorname{CRs}\left(t_{(8)}=3.6 ; p=0.008\right)$.

Figure 3 shows the temporal profiles from the activated volumes in V1 averaged across subjects for each paradigm at the four stages of the experiment. In contrast to the pattern observed in the activated volume, trace conditioning resulted in little change in the maximum BOLD magnitude across the stages of the experiment, with mean responses of $1.2 \pm 0.25 \%$ during pseudoconditioning CS trials, $1.5 \pm 0.11 \%$ at $40 \%$ CRs, $1.4 \pm 0.12 \%$ at $80 \%$ CRs, and $1.3 \pm 0.29 \%$ during memory trials. The mean BOLD response from the delay subjects, in contrast, exhibited significant changes with training, with a mean response of $1.8 \pm 0.33 \%$ during pseudoconditioning CS trials, versus $3.3 \pm 0.39 \%$ at $40 \%$ CRs, $4.2 \pm 0.69 \%$ at $80 \%$ CRs, and $2.7 \pm 0.46 \%$ during memory trials. Also note the extended elevation of the BOLD signal over time with respect to the baseline after the initial peak at 40 and $80 \%$ CRs for delay subjects.
A summary of the mean peak magnitude of the BOLD responses for the trace and delay subjects at each stage of the experiment is shown in Figure 4. A repeated-measures ANOVA with paradigm as a factor revealed a significant interaction $\left(F_{(2,16)}=\right.$ $5.02 ; p=0.020$ ) between the training paradigm (trace/delay) and the three stages of training (pseudoconditioning, 40 and $80 \%$ ). A post hoc ANOVA for each group separately indicated that the delay-conditioned group exhibited a significant difference $\left(F_{(2,8)}\right.$ $=7.78 ; p=0.013)$ among the three stages, but the traceconditioned group did not. Post hoc Fisher's LSD tests among the delay-conditioned rabbits indicated that the difference occurred between the baseline pseudoconditioning session and both the session at $40 \%$ CRs and at $80 \%$ CRs ( $p=0.04$ and 0.005 , respectively). Post hoc $t$ tests were used to compare the peak amplitude of the BOLD response between delay and trace conditioning at each stage of the experiment. These comparisons revealed a significant increase in the peak BOLD response for delayconditioned subjects relative to trace-conditioned subjects at $40 \% \operatorname{CRs}\left(t_{(8)}=4.6 ; p=0.002\right), 80 \% \operatorname{CRs}\left(t_{(8)}=3.9 ; p=0.004\right)$, and the post-training memory test $\left(t_{(8)}=2.5 ; p=0.039\right)$; there was no significant difference between the groups during the CS alone pseudoconditioning trials.

Two subcortical components of the visual system, the lateral geniculate nucleus (LGN) and the superior colliculus, were also examined on the side contralateral to the stimulus presentation. However, no active voxels were observed in these regions at any stage of the experiment.

To identify any learning-related changes in the sensory cortex mediating perception of the US, the eye region of the primary somatosensory cortex was analyzed on the side contralateral to the US presentation in the same manner as the primary visual cortex. Functional activation was observed in this region for both delay and trace subjects during pseudoconditioning US trials and both stages of training, but not during pseudoconditioning CS or memory trials. For delay subjects, the activated volumes in this region were $3.4 \pm 1.1 \mathrm{~mm}^{3}$ during pseudoconditioning US trials, $2.7 \pm 1.5 \mathrm{~mm}^{3}$ at $40 \% \mathrm{CRs}$, and $3.7 \pm 1.5 \mathrm{~mm}^{3}$ at $80 \% \mathrm{CRs}$, and the maximum BOLD responses were $1.9 \pm 0.40 \%, 2.3 \pm 0.56 \%$, and $2.1 \pm 0.39 \%$ for pseudoconditioning US trials, $40 \%$ CRs, and $80 \%$ CRs, respectively. For trace subjects, the activated volumes in this region were $3.3 \pm 1.3 \mathrm{~mm}^{3}$ during pseudoconditioning US trials, $4.4 \pm 0.50 \mathrm{~mm}^{3}$ at $40 \% \mathrm{CRs}$, and $4.4 \pm 1.2 \mathrm{~mm}^{3}$ at $80 \%$ CRs, and the maximum BOLD responses were $1.3 \pm 0.24 \%$, $1.7 \pm 0.31 \%$, and $2.2 \pm 0.27 \%$ for pseudoconditioning US trials, $40 \%$ CRs, and $80 \%$ CRs, respectively. A repeated-measures ANOVA with paradigm as a factor revealed no significant difference $(p>0.05)$ between the delay and trace paradigms in terms of either activated volume or maximum BOLD response, and $t$ tests performed within each paradigm showed no significant learning-related change between pseudoconditioning US trials and the training stages $(p>0.05)$.

\section{Discussion}

Our results demonstrate that delay and trace conditioning produce distinct learning-related changes in V1. During delay conditioning, the activated volume within V1 undergoes little change with training, whereas the BOLD response magnitude more than doubles during early and late learning. In contrast, activated volume significantly increases early in learning during trace conditioning, whereas the BOLD response magnitude remains relatively unchanged.

The difference in the neuronal substrates required to learn these paradigms is well established. Decorticate animals can ac- 

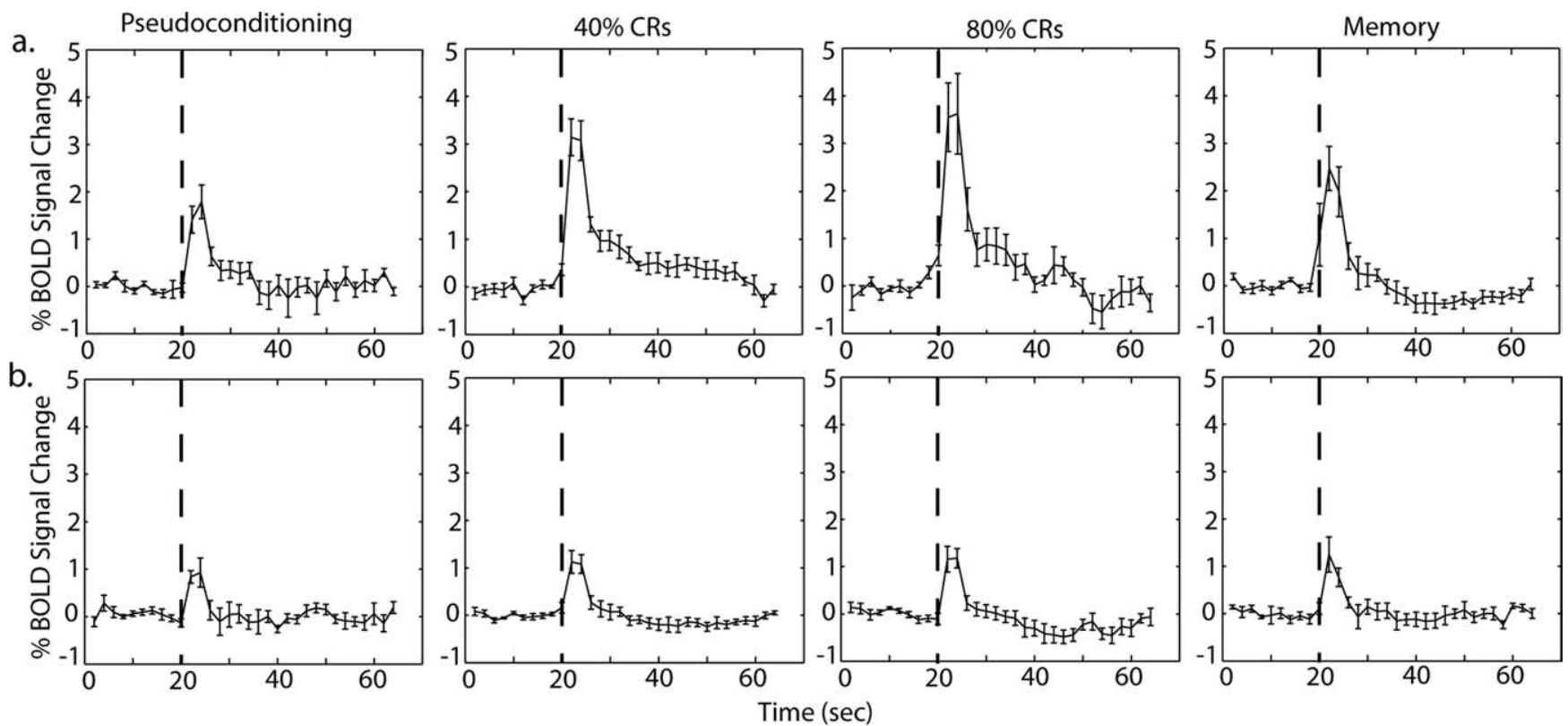

Figure 3. Averaged BOLD responses in V1. $\boldsymbol{a}, \boldsymbol{b}, \mathrm{BOLD}$ response from activated voxels within V1 averaged across subjects $(n=5$ for each paradigm) for delay $(\boldsymbol{a})$ and trace $(\boldsymbol{b})$ conditioning. Note the increase in magnitude in delay subjects during training ( 40 and $80 \%$ CRs) compared with pseudoconditioning and the subsequent decrease during memory trials. In contrast, the BOLD response undergoes little change during trace conditioning. The dashed line indicates the stimulus presentation. Error bars represent SEM.

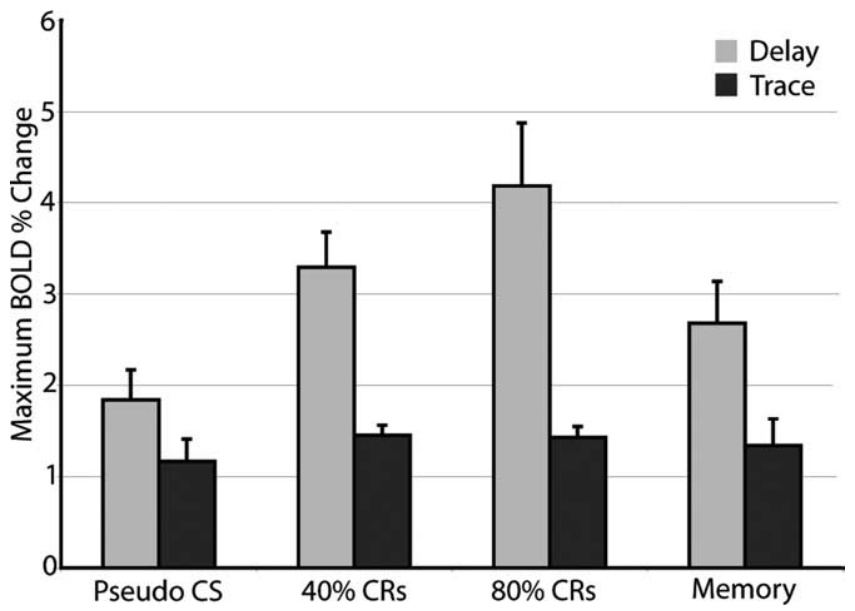

Figure 4. Maximum BOLD magnitude in V1. The mean ( $n=5$ for each paradigm) maximum magnitude of the BOLD response for both delay and trace conditioning is shown during pseudoconditioning CS trials, at 40 and $80 \%$ CRs, and during memory trials. Note that trace conditioning produces no significant change in maximum BOLD response over the course of the experiment, whereas delay conditioning produces a significantly elevated response $(p<0.05)$ with respect to pseudoconditioning CS trials at 40 and $80 \%$ CRs and with respect to trace conditioning at $40 \%$ CRs, $80 \%$ CRs, and memory stages. Error bars represent SEM.

quire delay-conditioned responses (Koutaldis et al., 1988), and decerebrate animals learn and retain delay conditioning (Norman et al., 1977; Mauk and Thompson, 1987), whereas trace conditioning requires intact forebrain structures. For example, lesions of the hippocampus (Solomon et al. 1986; Moyer et al. 1990), anterior cingulate cortex (Weible et al., 2000), and whisker barrel cortex (Galvez et al., 2007) can impair the acquisition of trace conditioning. Fundamentally, this difference must lie in the presence of the trace interval. The need to process this additional temporal information increases the cognitive difficulty of the trace paradigm and requires the engagement of a more complex circuit. Because V1 mediates the perception of the CS, which changes its behavioral significance during the experiment, it is expected that this region would exhibit learning-related changes, and that these changes could reflect the difference between conditioning paradigms with different levels of forebrain dependence.

Our results indicate that this difference in cognitive difficulty between the trace and delay paradigms translates to a differential level of support for the learned association within V1 during their acquisition. In the simpler, forebrain-independent delay paradigm the neurons exhibit an increase in response with no accompanying increases in activated volume by the time they attain a performance level of at least $40 \%$ CRs. The duration of the BOLD response also increases for delay subjects during training, a pattern that we observed in the cerebellar cortex and deep nuclei in our previous fMRI study of delay EBC. In contrast, the trace paradigm appears to require the recruitment of additional neurons to support the increased cognitive demand, which would account for the increase in activated volume, without any accompanying increase in the mean amplitude or duration of the BOLD response. Notably, these changes occur with trace conditioning even though the CS is shorter in duration $(250 \mathrm{~ms}$, vs $850 \mathrm{~ms}$ for delay). However, on the scale of the time resolution of the fMRI sequence (i.e., $2 \mathrm{~s}$ ), both stimuli are essentially "impulses," and no significant difference was observed between the stimuli during pseudoconditioning CS trials in terms of either activated volume or peak BOLD response magnitude.

The stage of the experiment at which this change in activated volume occurs could reflect the role of V1 in both the initial formation and longer-term storage of the learned association. Our results indicate that this increase in activated volume in V1 occurred by the midpoint of trace conditioning and remained elevated from baseline in well trained animals and during CSalone memory trials. Experiments recording neuronal activity in the hippocampus during trace EBC suggest that the pattern of changes in the hippocampus likely precede those we observed in the V1 BOLD response because the initial alterations in firing rate occur before the onset of CRs during training and are well established by the time rabbits show $40 \%$ CRs (McEchron and Dister- 
hoft, 1997; Weible et al., 2006). The anterior cingulate cortex exhibited an increase in excitatory neuronal responses even earlier in the trace conditioning trial sequence than the hippocampus, followed by a reduction in firing rate by the stage in learning that significant CRs became evident (Weible et al., 2003). The patterns in these regions suggest that V1 plays an active role during the formation and storage of the learned association during trace conditioning, in contrast to the hippocampus and anterior cingulate which are predominantly active during the initial formation of the memory, with hippocampal activity remaining at the points in the learning sequence when we observed V1 BOLD responses. No such pattern of volume change was observed in V1 during delay conditioning, although this region did exhibit an increase in BOLD response magnitude that persisted throughout both stages of training, as well as CS-alone memory trials. Similarly, robust neuronal responses have been observed in the hippocampus during delay conditioning (Berger et al., 1983), and the responses were greater in terms of amplitude and duration during delay than during trace conditioning (Weiss et al., 1996) even though this region, like $\mathrm{V} 1$, is not required for acquisition of the paradigm.

Previous nonhuman animal studies investigating learningrelated plasticity in the primary sensory cortices have also detected changes characteristic of the paradigms involved, and have distinguished between increases in the responsiveness of cortical neurons and expansion of representational area. For example, fear conditioning paradigms (i.e., tone-shock pairing) have produced increases in neuronal response in the receptive fields of the primary auditory cortex (A1) corresponding to the CS frequency (Bakin and Weinberger, 1990; Edeline et al., 1993). Such changes can persist for weeks, supporting the assertion that such plasticity represents long-term storage of information in the cortex (Weinberger et al., 1993). These studies also showed decreases in the response of receptive fields corresponding to adjacent frequencies. More short-term changes have also been shown in the spectrotemporal receptive fields of A1 using auditory discrimination training (Fritz et al., 2003).

Similar results have also been found in human studies using functional imaging. PET studies have detected learning-related activation in A1 during both delay EBC (Molchan et al., 1994; Schreurs et al., 1997) and fear conditioning (Morris et al., 1998). An fMRI study of fear conditioning using a delay paradigm (Knight et al., 1999) revealed an increased BOLD response in the visual cortex, with no increase in the area of activation in the paired-trial group. Similar increases in functional activity were also observed in the visual cortex using fMRI during fear conditioning (Cheng et al., 2003).

Learning-related expansion of representation within the primary sensory cortices has also been demonstrated. Cytochromeoxidase staining has revealed expansion of the whisker barrels after trace EBC with a whisker-vibration CS (Galvez et al., 2006). Gruart et al. (2000) demonstrated that both trace and delay EBC resulted in enhanced c-Fos expression in the auditory cortex after training to a criterion level of $85 \%$ CRs. Interestingly, their study observed no significant difference between trace and delay conditioning at this late stage of learning, which agrees with our findings. Electrophysiological recordings in the auditory cortex during delay EBC (Kraus and Disterhoft, 1982), operant conditioning (Rutkowski and Weinberger, 2005), and discrimination training (Recanzone et al., 1992) have revealed CS-specific representational expansion. Tactile discrimination training has also produced enlarged representation in S1 (Recanzone et al., 1993). Pleger et al. (2003) observed a similar enlargement of cortical representation in S1 related to discrimination training in a human fMRI study.

Thus, significant precedent exists for increases in both neuronal responsiveness and representational area as distinct manifestations of learning-related cortical plasticity. However, direct comparison between our results and much of the previous work is complicated by the wide variety of animal preparations, learning paradigms, and stimulus presentations that have been used. As described previously, several studies of CS-specific receptive field tuning detected both increases and decreases in receptive field response, whereas we observed only an increase in BOLD response. One consideration is that our study used a nonspecific visual stimulus, in terms of receptive field activation, in contrast to the clearly defined frequencies used in studies of the auditory cortex. Furthermore, our voxel size is larger than the regions measured by recording electrodes, and thus represents the averaged response of a larger assembly of neurons. However, if a more complex pattern of neuronal response is present below the resolution of fMRI, our results suggest that the overall response is positive.

One important consideration in interpreting learning-related changes in the cortex is whether the changes reflect intracortical plasticity, modulation by other structures in the circuit, such as the thalamic nuclei, or a combination of these effects. Notably, no activity was detected in either the LGN or superior colliculus at any stage during these experiments, although a number of previous studies have demonstrated a role for the subcortical structures of the sensory systems in classical conditioning. Lesions of the auditory (LeDoux et al., 1986; Halverson and Freeman, 2006) and visual (Koutaldis et al., 1988) thalamic nuclei have been shown to inhibit classical conditioning. Electrophysiological recordings have demonstrated that classical conditioning induces retuning of the auditory thalamic nuclei (Edeline and Weinberger, 1992). Gonzalez-Lima and Scheich (1984) detected learning-related metabolic changes in the auditory nuclei during classical conditioning using 2-deoxyglucose autoradiography; however, they observed no changes in the metabolic effects of the CS before and after conditioning in the medial geniculate nucleus, and suggest that activity in this structure is more reflective of the behavioral arousal of the US. One factor that could have influenced the absence of any observed BOLD activity in subcortical visual structures in our study is the nature of the CS used in our experiments. An fMRI study of the frequency dependence of the rat visual system (Van Camp et al., 2006) found that BOLD response in the superior colliculus was greatest at higher frequencies, and reduced at low frequencies. In contrast, the visual cortex showed no such reduction in BOLD response at low frequencies. Thus, the continuous visual stimulus used in this study may have been better suited to producing cortical activation. Notably, we also used a less aversive US (i.e., a corneal airpuff vs a foot shock), as well as a different animal preparation and behavioral model. However, our results suggest that the learning-related changes observed during delay and trace conditioning do not merely reflect activity in antecedent structures within the visual system. Likewise, although activity was present consistently in S1 during pseudoconditioning US trials and training, no learning related change in activated volume or magnitude of the temporal profiles was observed. This result suggests that any potential contribution of S1, via intracortical projections, to the learning-related changes observed in V1 is minimal.

Although our results indicate that neither the visual thalamic nuclei nor the somatosensory cortex were dynamically involved in the learning-related changes associated with visual cued EBC, 
other forebrain regions could also potentially modulate V1. For example, cholinergic input from the nucleus basalis (NB) has been shown to facilitate cortical plasticity (Rasmusson and Dykes, 1988; Kilgard and Merzenich, 1998; Bao et al., 2001; McLin et al., 2002). Projections from striatal structures such as the caudate nucleus could also potentially modulate cortical activity. The caudate receives projections from a large number of cortical regions including V1, and previous electrophysiological (White et al., 1994) and lesion (Powell et al., 1978) studies have indicated that activity in this structure affects the formation of CRs. Future analysis of responses in the NB and striatum should provide a clearer picture of their respective contributions to learning-related plasticity.

In summary, our results indicate that the difference in cognitive difficulty between trace and delay conditioning is reflected by the pattern of learning-related functional activation within V1. Delay conditioning produced a strengthening of the BOLD response but no accompanying increase in volume. In contrast, the expansion of the activated volume during forebrain-dependent trace conditioning suggests that the recruitment of additional neurons, particularly early in learning, is necessary to mediate the formation of the more complex learned association. Furthermore, the absence of either activation within the thalamic nuclei of the visual system or learning-related change in S1 suggests that the functional changes in V1 do not merely reflect activity from antecedent visual areas and are not significantly influenced by input from other sensory cortical regions. This work represents the first functional imaging study to compare directly the responses of delay versus trace conditioning in V1. However, examination of the hippocampus, as well as other forebrain structures such as the NB and caudate nucleus, should provide a more comprehensive understanding of the relative contributions of these regions to memory formation.

\section{References}

Bakin JS, Weinberger NM (1990) Classical conditioning induces CSspecific receptive field plasticity in the auditory cortex of the guinea pig. Brain Res 536:271-286.

Bao S, Chan VT, Merzenich MM (2001) Cortical remodeling induced by activity of ventral tegmental dopamine neurons. Nature 412:79-83.

Berger TW, Rinaldi P, Weisz DJ, Thompson RF (1983) Single unit analysis of different hippocampal cell types during classical conditioning of the rabbit. J Neurophysiol 50:1197-1219.

Cheng DT, Knight DC, Smith CN, Stein EA, Helmstetter FJ (2003) Functional MRI of human amygdyla activity during Pavlovian fear conditioning: stimulus processing versus response expression. Behav Neurosci 117:3-10.

Chihara E (1982) Reexamination of decussated fibers at the optic chiasma of rabbits. Graefes Arch Clin Exp Ophthal 218:28-29.

Choudhury BP (1987) Visual-cortex in the albino rabbit. Exp Brain Res 66: $565-571$.

Edeline JM, Weinberger NM (1992) Associative retuning in the thalamic source of input to the amygdyla and auditory cortex: receptive field plasticity in the medial division of the medial geniculate body. Behav Neurosci 106:81-105.

Edeline JM, Pham P, Weinberger NM (1993) Rapid development of learning-induced receptive field plasticity in the auditory cortex. Behav Neurosci 107:539-557.

Fritz JB, Shamma SA, Elhilali M, Klein DJ (2003) Rapid task-related plasticity of spectrotemporal receptive fields in primary auditory cortex. Nat Neurosci 6:1216-1223.

Galvez R, Weiss C, Weible AP, Disterhoft JF (2006) Vibrissa-signaled eyeblink conditioning induces somatosensory cortical plasticity. J Neurosci 26:6062-6068.

Galvez R, Weible AP, Disterhoft JF (2007) Cortical barrel lesions impair whisker-CS trace eyeblink conditioning. Learn Mem 14:94-100.
Giolli RA, Guthrie MD (1969) Primary optic projections in rabbit. An experimental degeneration study. J Comp Neurol 136:99-125.

Gonzalez-Lima F, Scheich H (1984) Neural substrates for tone-conditioned bradycardia demonstrated with 2-deoxyglucose. I. Activation of auditory nuclei. Behav Brain Res 14:213-233.

Gormezano I, Kehoe EJ, Marshall BS (1983) Twenty years of classical conditioning research with the rabbit. Prog Psychobiol Physiol Psychol 10:197-275.

Gruart A, Morcuende S, Martinez A, Delgado-Garcia JM (2000) Involvement of cerebral cortical structures in the classical conditioning of eyelid responses in rabbits. Neurosci 100:719-730.

Halverson HE, Freeman JH (2006) Medial auditory thalamic nuclei are necessary for eyeblink conditioning. Behav Neurosci 120:880-887.

Hughes A, Vaney DI (1982) The organization of binocular cortex in the primary visual area of the rabbit. J Comp Neurol 204:151-164.

Ibanez L, Schroeder W (2005) The ITK software guide 2.4. Clifton Park, NY: Kitware.

Kaas JH, Krubitzer LA, Chino YM, Langston AL, Polley EH, Blair N (1990) Reorganization of retinotopic cortical maps in adult mammals after lesions of the retina. Science 248:229-231.

Kilgard MP, Merzenich MM (1998) Cortical map reorganization enabled by nucleus basalis activity. Science 297:1714-1718.

Knight DC, Smith CN, Stein EA, Helmstetter FJ (1999) Functional MRI of human Pavlovian fear conditioning: patterns of activation as a function of learning. NeuroReport 10:3665-3670.

Koutaldis O, Foster A, Weisz DJ (1988) Parallel pathways can conduct visual $\mathrm{CS}$ information during classical conditioning of the NM response. J Neurosci 8:417-427.

Kraus N, Disterhoft JF (1982) Response plasticity of single neurons in rabbit auditory cortex during tone-signalled learning. Brain Res 246:205-215.

Lavond DG, Kim JJ, Thompson RF (1993) Mammalian brain substrates of aversive classical conditioning. Annu Rev Psychol 44:317-342.

LeDoux JE, Iwata J, Pearl D, Reis DJ (1986) Disruption of auditory but not visual learning by destruction of intrinsic neurons in the medial geniculate body of the rat. Brain Res 371:395-399.

Li L, Weiss C, Disterhoft JF, Wyrwicz AM (2003) Functional magnetic resonance imaging in the awake rabbit: a system for stimulus presentation and response detection during eyeblink conditioning. J Neurosci Methods 130:45-52.

Mauk MD, Thompson RF (1987) Retention of classically conditioned eyelid responses following acute decerebration. Brain Res 493:89-95.

McEchron MD, Disterhoft JF (1997) Sequence of single neuron changes in CA1 hippocampus of rabbits during acquisition of trace eyeblink conditioned responses. J Neurophysiol 78:1030-1044.

McLin DD, Miasnikov AA, Weinberger NM (2002) Induction of behavioral associative memory by stimulation of the nucleus basalis. Proc Natl Acad Sci USA 99:4002-4007.

Merzenich MM, Nelson RJ, Stryker MP, Cynader MS, Schoppmann A, Zook JM (1984) Somatosensory cortical map changes following digit amputation in adult monkeys. J Comp Neurol 224:591-605.

Miller MJ, Chen NK, Li L, Tom B, Weiss C, Disterhoft JF, Wyrwicz AM (2003) fMRI of the conscious rabbit during unilateral classical eyeblink conditioning reveals bilateral cerebellar activation. J Neurosci 23:11753-11758.

Miller MJ, Li L, Weiss C, Disterhoft JF, Wyrwicz AM (2005) A fiber opticbased system for behavioral eyeblink measurement in a MRI environment. J Neurosci Methods 141:83-87.

Mitra P, Pesaran B (1999) Analysis of dynamic brain imaging data. Biophys J 76:691-708.

Molchan SE, Sunderland T, McIntosh AR, Herscovitch P, Schreurs BE (1994) A functional anatomical study of associative learning in humans. Proc Natl Acad Sci USA 91:8122-8126.

Morris JS, Friston KJ, Dolan RJ (1998) Experience-dependent modulation of tonotopic neural responses in human auditory cortex. Proc Biol Sci 265:649-667.

Moyer JR, Deyo RA, Disterhoft JF (1990) Hippocampectomy disrupts trace eye-blink conditioning in rabbits. Behav Neurosci 104:243-252.

Murphy EH, Berman N (1979) The rabbit and the cat: a comparison of some features of response properties of single cells in the primary visual cortex. J Comp Neurol 188:401-428.

Norman RJ, Buchwald JS, Villiblanca JR (1977) Classical conditioning with 
auditory discrimination of the eyeblink in decerebrate cats. Science 196:551-553.

Pleger B, Foerster AF, Ragert P, Dinse HR, Schwenkreis P, Malin JP, Nicolas V, Tegenthoff M (2003) Functional imaging of perceptual learning in human primary and secondary somatosensory cortex. Neuron 40:643-653.

Powell DA, Mankowski D, Buchanan S (1978) Concomitant heart-rate and corneoretinal potential conditioning in rabbit (Oryctolagus cuniculus): effects of caudate lesions. Physiol Behav 20:143-150.

Rasmusson DD, Dykes RW (1988) Long-term enhancement of evoked potentials in cat somatosensory cortex produced by co-activation of basal forebrain and cutaneous receptors. Exp Brain Res 70:276-286.

Recanzone GH, Merzenich MM, Schreiner CE (1992) Changes in the distributed temporal response properties of SI cortical neurons reflect improvements on a temporally based tactile discrimination task. J Neurophysiol 67:1071-1090.

Recanzone GH, Schreiner CE, Merzenich MM (1993) Plasticity in the frequency representation of primary auditory cortex following discrimination training in adult owl monkeys. J Neurosci 13:87-103.

Rutkowski RG, Weinberger NM (2005) Encoding of learned importance of sound by magnitude of representational area in primary auditory cortex. Proc Natl Acad Sci USA 102:13644-13669.

Schreurs BG, Alkon DL (2001) Imaging learning and memory: classical conditioning. Anat Rec 265:257-273.

Schreurs BG, McIntosh AR, Bahro M, Herscovitch P, Sunderland T, Molchan SE (1997) Lateralization and behavioral correlation of changes in regional cerebral blood flow with classical conditioning of the human eyeblink response. J Neurophysiol 77:2153-2163.

Solomon PR, Vander Schaaf ER, Thompson RF, Weisz DJ (1986) Hippocampus and trace conditioning of the rabbit's classically conditioned nictitating membrane response. Behav Neurosci 100:729-744.

Song X, Murphy M, Wyrwicz AM (2006) Spatiotemporal denoising and clustering of fMRI data. Proc IEEE Conf Image Process 2006:2857-2860.

Song X, Iordanescu G, Wyrwicz AM (2007) One-class machine learning for brain activation detection. Proc IEEE Conf Comput Vis Pattern Recog 2007:1-6.

Steele RI, van Hof MW, van der Steen J, Collewijn H (1987) Visual and oculomotor function in optic chiasma-sectioned rabbits. Exp Brain Res 66:61-73.

Thompson JM, Woolsey CN, Talbot SA (1950) Visual areas I and II of cerebral cortex of rabbit. J Neurophysiol 13:277-288.

Thompson RF, Kim JJ (1996) Memory systems in the brain and localization of a memory. Proc Natl Acad Sci USA 93:11438-11444.

Van Camp N, Verhoye M, De Zeeuw CI, Van der Linden A (2006) Light stimulus frequency dependence of activity in the rat visual system as studied with high-resolution BOLD fMRI. J Neurophysiol 95:3164-3170.

Weible AP, McEchron MD, Disterhoft JF (2000) Cortical involvement in acquisition and extinction of trace eyeblink conditioning. Behav Neurosci 114:1058-1067.

Weible AP, Weiss C, Disterhoft JF (2003) Activity profiles of single neurons in caudal anterior cingulate cortex during trace eyeblink conditioning in the rabbit. J Neurophysiol 90:599-612.

Weible AP, O'Reilley JA, Weiss C, Disterhoft JF (2006) Comparisons of dorsal and ventral hippocampus cornu ammonis region 1 pyramidal neuron activity during trace eye-blink conditioning in the rabbit. Neuroscience 141:1123-1137.

Weinberger NM, Javid R, Lepan B (1993) Long-term retention of learninginduced receptive-field plasticity in the auditory cortex. Proc Natl Acad Sci USA 90:2394-2398.

Weiss C, Kronforst-Collins MA, Disterhoft JF (1996) Activity of hippocampal pyramidal neurons during trace eyeblink conditioning. Hippocampus 6:192-209.

Weiss C, Weible AP, Galvez R, Disterhoft JF (2006) Forebrain-cerebellar interactions during learning. Cell Sci Rev 3:200-231.

White IM, Miller DP, White W, Dike GL, Rebec DV, Steinmetz JE (1994) Neuronal activity in rabbit neostriatum during classical eyelid conditioning. Exp Brain Res 99:179-190.

Wyrwicz AM, Chen NK, Li L, Weiss C, Disterhoft JF (2000) fMRI of visual system activation in the conscious rabbit. Magn Reson Med 44:474-478. 